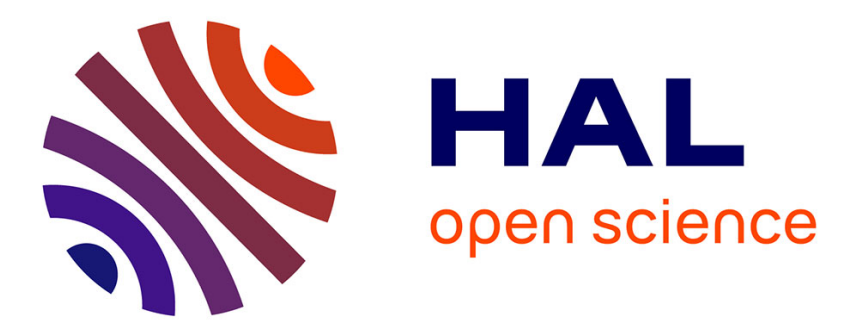

\title{
Wire-bond contact degradation modeling for remaining useful lifetime prognosis of IGBT power modules
}

\author{
M Nazar, Ali Ibrahim, Zoubir Khatir, Nicolas Degrenne, Z Al-Masry
}

\section{To cite this version:}

M Nazar, Ali Ibrahim, Zoubir Khatir, Nicolas Degrenne, Z Al-Masry. Wire-bond contact degradation modeling for remaining useful lifetime prognosis of IGBT power modules. Microelectronics Reliability, 2020, 114, 10.1016/j.microrel.2020.113824 . hal-03150944

\author{
HAL Id: hal-03150944 \\ https://hal.science/hal-03150944
}

Submitted on 24 Feb 2021

HAL is a multi-disciplinary open access archive for the deposit and dissemination of scientific research documents, whether they are published or not. The documents may come from teaching and research institutions in France or abroad, or from public or private research centers.
L'archive ouverte pluridisciplinaire $\mathbf{H A L}$, est destinée au dépôt et à la diffusion de documents scientifiques de niveau recherche, publiés ou non, émanant des établissements d'enseignement et de recherche français ou étrangers, des laboratoires publics ou privés. 


\title{
Wire-bond Contact Degradation Modelling for Remaining Useful Lifetime Prognosis of IGBT Power Modules
}

\author{
M. Nazar, A. Ibrahim, Z. Khatir, N. Degrenne, Z. Al-Masry
}

\begin{abstract}
Reliability of power electronic equipment can be enhanced with the ability to predict its health state to avoid damage and permit making necessary maintenance. In this paper, an analytical physics of failure model is built that describes the contact resistance between wire-bonds and pad-metallization in an IGBT semiconductor device. On one hand, this model is based on the theory of the electrical contact resistance and the other hand on the spread of the current from the contact to the aluminum film. When contact degradations occur, the model can relate the crack propagation in the wire-bond interconnection to the collectoremitter voltage $\left(V_{C E}\right)$ and may allow identifying the health of the device. If the voltage is measured during actual operation, the length of the contact radius can be estimated and crack propagation rate can be calculated and used to predict the remaining useful life. The approach is tested with experimental accelerated aging tests.
\end{abstract}

\section{Introduction}

The reliability of power electronic devices is an important issue for most power electronic systems. Electric components such as IGBTs are subject to high environmental and functional stresses (ambient temperature, vibrations...). All these factors have a strong impact on device reliability and lifetime.

Prediction of the Remaining Useful Lifetime (RUL) with a high confidence level is needed to assure reliability. It is important economically to have predictive maintenance for such devices than having preventive maintenance performed at fixed time intervals. One way to have a prediction approach is to have a model that describes the degradation in the power device. Two main kinds of models are presented in the literature for prognostic: the damage accumulation models and the condition based prediction models. The damage accumulation models can estimate the lifetime of components operating under given usage conditions. The effect of past usage is explicitly modeled by summing up the life consumption due to each stress undergone by the component in operation [1,2]. Research is ongoing to improve these types of models, for example, N. Dornic [3] gives the dependence of the $N_{f}$ (number of cycles until failure) with the stress duration $t_{o n}$ and junction temperature swing amplitudes $\Delta T_{j}$. On the other hand, condition-based prediction models are based on the measurement and extrapolation of a precursor to failure. It assesses the behavior of individual units not only on their usage history but also on expected future load profiles. In other words, it utilizes a degradation model that predicts the future state based on input that describes the current system state and the expected load levels on the system. These component-specific models are generally adaptable and can be developed based on the physics of the system like[4,5], or can be learned from run to failure data using data-driven methods like [6,7]. Sreenuch in [6] used a probabilistic Monte-Carlo method for modeling and online prediction of the remaining useful life (RUL) in an electronic component. His study is based on the $V_{C E}$ data collected from the electronic component, also Zhen Liu in [8] used the Machine learning method to predict RUL for IGBT semiconductors using degradation data.

The remainder of the paper is organized as follows. Section 2 defines different types of degradations that occur in IGBT devices due to aging. Section 3 presents the analytical model used for RUL prediction. It describes the evolution of the dominant degradation that takes place in the studied module. It is based on the theory of contact resistance and on the spreading resistance in a thin metallization film. In section 4 , an online prognostics model methodology is presented to predict the RUL. The steps for building the model are illustrated. This model is tested on experimental results with different stress conditions.

\section{Aging behaviors and Degradations}

During the aging of power electronic devices, different types of degradations can take place as shown in Fig.1.

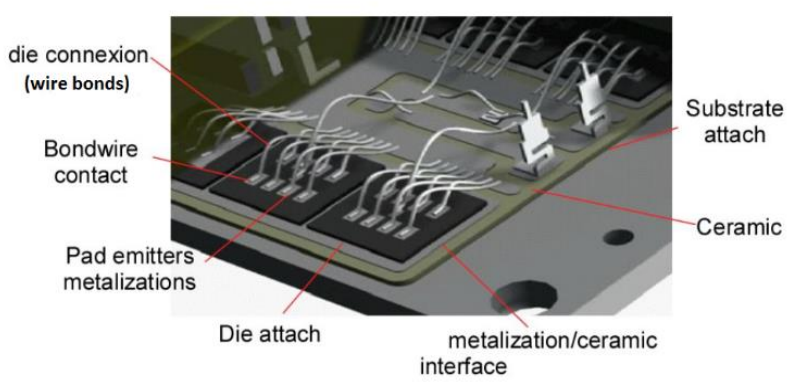

Fig.1: Possible degradation location in IGBT power module

The degradation types are: wire-bond degradation that usually leads to heel cracks, wire-bond lift-off due to crack propagation in the wire-bond interconnection with the metallization, top metallization degradation due to grains recrystallization, die-attach degradation that leads to cracks at the die bottom connection, cracks in the metallization ceramic interface, cracks in the ceramic itself, and substrate attach that leads to solder cracks.

In the IGBT module, wire-bond lift-off is a dominant degradation (see Fig.2a[9]). For this reason, the focus in this paper is related to the crack propagation leading to the lift-off. (Fig.2b) presents crack propagation in the wire-bond interconnection with the metallization.. 


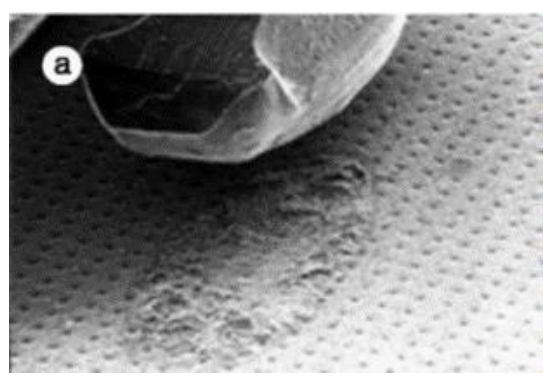

(b)

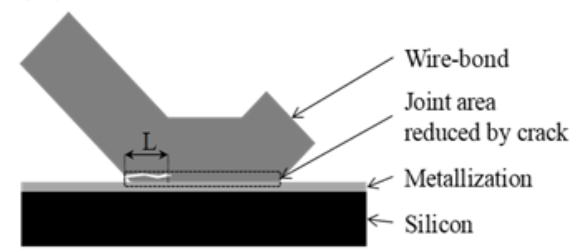

Fig.2: a) wire-bond lift-off [9], b) crack propagation in the wire-bond interconnection with the metallization [4]

A way to observe the $V_{C E}$ evolution with degradation due to thermal fatigue is using accelerated aging tests like power cycling. Fig. 3 shows experimental data for 4 IGBT modules of the same type aged under the same stress conditions. As data from the field are not easy to obtain, these types of accelerated results are used hereafter for model testing and validation.

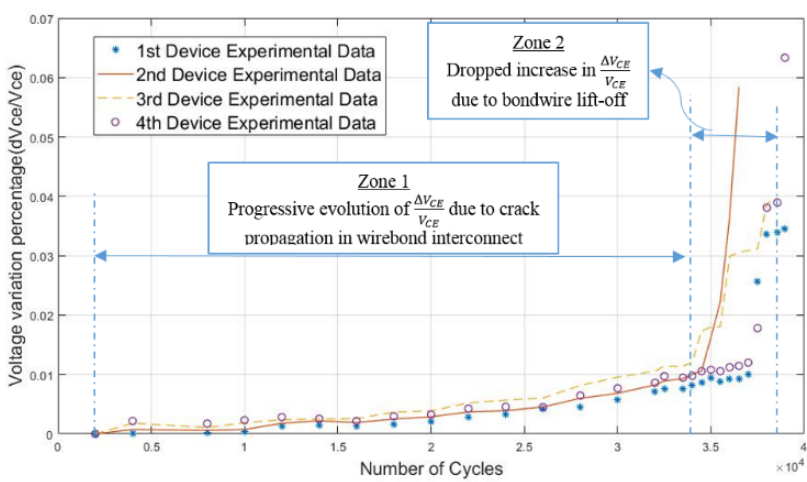

Fig.3: Collector-Emitter voltage variation, and its zones due to types of degradation

The only degradation observed in these aging tests is the lift-off due to crack propagation. Hence, it is assumed that the voltage variation in the wire-bond contact is the only responsible of the voltage variation observed between the collector and the emitter terminals.

In Fig.3, the experimental accelerated conditions used are from power pulses with $3 \mathrm{~s}$ heating time and $6 \mathrm{~s}$ cooling time with power value that leads to $110^{\circ} \mathrm{C}$ temperature variation, from $35^{\circ} \mathrm{C}$ to $145^{\circ} \mathrm{C}$. This figure gives the $V_{C E}$ evolution during power cycling of the 4 devices under tests (DUTs). The lifetime of the devices can be divided into two main zones. In zone 1, the voltage increase is due to the crack propagation in the wire-bond interconnection with the metallization. At the end of this zone, more than $80 \%$ of the lifetime is passed. Zone 2 presents the increase in voltage due to the lift-off process. The focus of this paper is related to the degradation in zone 1.

\section{Contact Resistance Physical Model}

In this section, an analytical model is built that estimates the increase of the crack length at the wire-bond contact from the increase in the $V_{C E}$. This model is then used to predict the RUL, assuming a mean speed linear crack propagation. In comparison to literature, a study done by Degrenne and Mollov [4] presents a physical model that relates the crack propagation to on-state voltage. Using a specific algorithm, this model is used to predict the RUL with an accuracy of less than $\pm 10 \%$. This model assumed that crack propagation is linear. However, $\mathrm{Hu}$ in [5] built a physical model that relates the crack propagation to the voltage using the Finite Element Method and taking into consideration nonlinear crack propagation. This model took into account also the effects of temperature variation and metallization degradation on the on-state voltage increase.

The model in this paper combines two different models. The first one describes the constriction resistance at the bond contact due to the crack from the wire-bond side. The second one gives the spreading resistance in the metallization due to the current lines flowing from the die, collected by the metallization pad and going through the bonding wire. The spreading of the current lines is also affected by the crack length. The model is simplified in order to consider a disc as a surface contact between a cylindrical bond-wire and the metallization as depicted in Fig.4.

\subsection{Constriction Resistance in the wire-bond part}

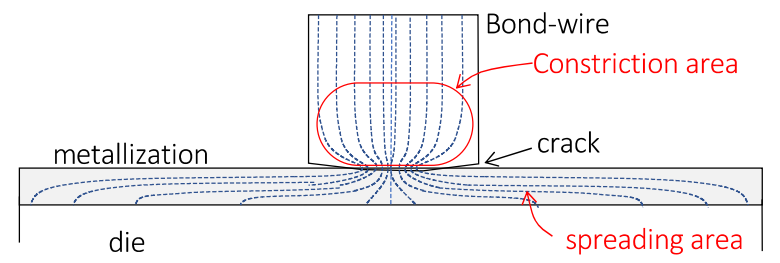

Fig.4: Model geometry with the constriction and the spreading areas.

Based on Timsit's relation [10], the constriction resistance in the wire-bond side is given by:

$$
\begin{gathered}
R_{C}=\frac{\rho}{4 a}\left[1-1.41581\left(\frac{a}{r}\right)+0.06322\left(\frac{a}{r}\right)^{2}+\right. \\
\left.0.15261\left(\frac{a}{r}\right)^{3}+0.19998\left(\frac{a}{r}\right)^{4}\right]
\end{gathered}
$$

where $\rho$ (resistivity of the material), $a$ (radius of the contact area), and $r$ is the redius of the wire-bond. This constriction resistance is due to the current constricted to pass through the contact area (see Fig.4). At first, when there is no crack and the contact is full, even if current lines are perturbed due to geometry, this is not strictly speaking a constriction zone.

According to Eq.1, $R_{C}$ is dependent on the radius of the area still in contact. Since crack propagates during the aging, the contact area decreases with the radius $a$ that leads to an increase in Constriction resistance $R_{C}$. 


\subsection{Metallisation's Resistance Model}

The resistance of the metallization is due to the lateral current flowing vertically from the die (see Fig.5). If the bondwire contact radius is $a$ and the radius of the considered metallization area is $b$, then the equivalent resistance of metallization, "seen" between $r=a$ and $r=b$ is given by eq.2, see appendix.

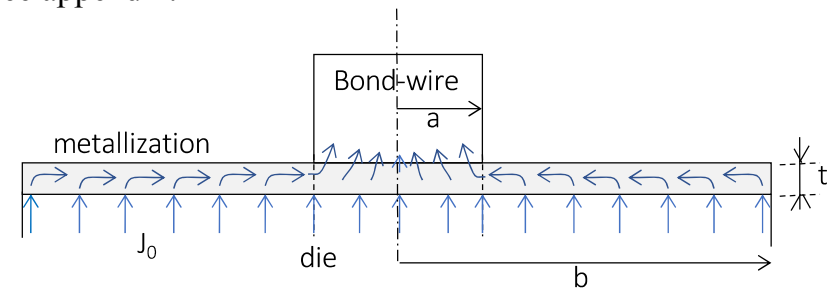

Fig.5: Current flow in the metallization

$$
R_{\text {met. }}=\frac{\rho}{2 \pi t}\left(\frac{b^{2}}{b^{2}-a^{2}} \ln \frac{b}{a}-\frac{1}{2}\right)
$$

Where $\mathrm{t}$ is the metallization thickness and $\rho$ its resistivity. The effect of crack propagation on the metallization resistance is very important to be modeled. It is shown later that the metallization resistance variation is high in comparison to the constriction resistance.

This resistance in Eq.2 covers the resistance of the metallization form $a$ to $b$. This relation doesn't include the resistance increase in the metallization due to resistivity increase and/or structure change (recrystallization and grain grooving). Nevertheless, the resistance of metallization increases with aging due to power cycling as mentioned in [11]. The literature reports that the metallization resistance can increase by a factor 2 to more than 10 depending on stress conditions [11-13]. Based on that, a factor " $w$ " is multiplied to the resistance of Eq.2 to form Eq.3. No additional measurements were done to fix " $w$ ", however, it is assumed to be constant and equal to 2 based on the study of [11] that carries almost the same conditions of power cycling tests used in this study.

$$
R=\frac{w \rho}{2 \pi t}\left(\frac{b^{2}}{b^{2}-a^{2}} \ln \left(\frac{b}{a}\right)-\frac{1}{2}\right)
$$

It is necessary to be mentioned that the wire-bond contact shape in IGBT is ellipsoidal. However, Holms mentioned in [14] that for ellipse contact shape the difference can be neglected if the multiplication of the ellipse's major and minor semi-axis is equal to $a^{2}$ (which is our situation). Moreover, the dimensions of the metallization are not circular; however, in this study " $b$ " is calculated experimentally by fitting the whole experimental curve to the model. Besides, a parameter sensitivity study for " $b$ " is done and it shows that changing $b$ in an acceptable range between $1.3 \mathrm{~mm}$ and $2 \mathrm{~mm}$ has a very small effect in changing the predicted curves

\subsection{Proposed model}

By combining two models described in 3.1 and 3.2, Eq.4 is built that describes the effect of crack propagation (contact area decrease) on the resistance between wire-bond and metallization.

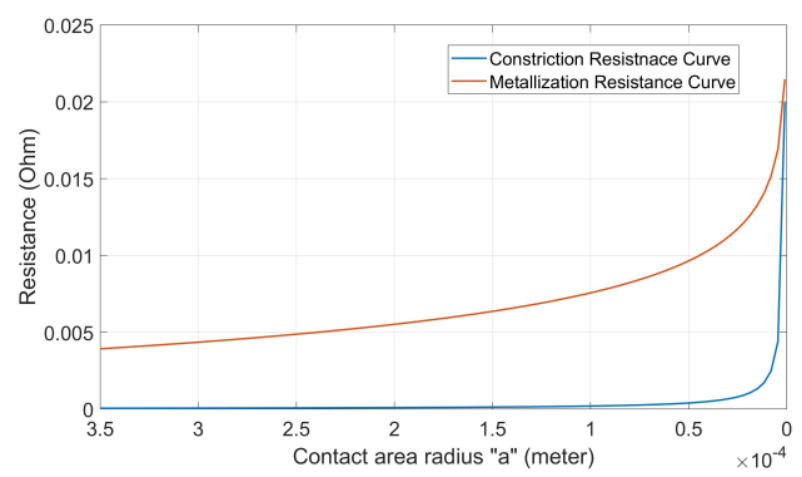

Fig.6: Effect of contact area radius "a" change in Eq. 1 \& 9 on the resistance

$$
\begin{gathered}
R(a)=\frac{\rho}{2}\left[\frac { 1 } { 2 a } \left(1-1.41581\left(\frac{a}{r}\right)+\right.\right. \\
0.06322\left(\frac{a}{r}\right)^{2}+0.15261\left(\frac{a}{r}\right)^{3}+ \\
\left.\left.0.19998\left(\frac{a}{r}\right)^{4}\right)+\frac{w}{\pi t}\left(\frac{b^{2}}{b^{2}-a^{2}} \ln \frac{b}{a}-\frac{1}{2}\right)\right]
\end{gathered}
$$

Fig. 6 shows the effect of the radius change of the bond contact on both resistances due to crack propagation. It is observed that with a decrease in the radius, the two resistances increase. It can be observed that the metallization resistance remains very high compared to that of constriction except when the contact is reduced to a very small radius. The sensitivity of resistance to radius change increases with radius decrease, and it increases in high slope at the last few micrometers.

The values of parameters of Eq. 1 \& 3 used in this study based on the structure are $b=2 \mathrm{~mm}$, radius initial value $a_{0}=$ $332 \mu m, t=4 \mu m$, and $\rho=3.8 * 10^{-8} \Omega m$

This model is used in the prognostics methodology to predict the remaining voltage variation at each measurement. Besides, as mentioned before, the variation of voltage calculated from Eq.3 equals the variation of collector-emitter voltage $\left(\Delta V_{C E}\right)$. The approach is more detailed in the next section.

\section{Prognostics Approach}

The prognostics approach for RUL estimation is based on Eq.4. At the current time, the $\Delta V_{C E} / V_{C E}$ value is measured (mth measurement), (see Fig.7). This is used to calculate the current contact area radius $a_{m}$ using Eqs.5-8 and compare it to the initial radius $a_{0}$. If a difference is detected, this means that a crack has occurred or progressed. The initial contact area radius $a_{0}$, as well as the successive contact radius $a_{i}(\mathrm{i}=1$ to $\mathrm{m})$ are used to compute the rate of crack propagation $\left(K_{m}\right)$, between the mth and the $(\mathrm{m}-1)$ th measurements, using Eq.9. For prognostic purpose, a mean value $\bar{K}$ of the successive $K_{m}$ is used to predict 
the evolution of the contact area radius with the assumption of linear crack propagation using Eq.10.

The detailed process is as follows, at first, relative variation in $V_{C E}$ is calculated, applying Ohm's law:

$$
\frac{\Delta V_{C E}}{V_{C E, \text { ini }}}=\frac{\Delta R(a)_{15 W} * I}{V_{C E, \text { ini }}}
$$

where $V_{C E, \text { ini }}$ is the initial collector-emitter voltage and $I$ is the direct current used. $\Delta R(a)_{15 W}$ is the resistance variation of 15 parallel wire-bonds connected to the metallization pad. Eleven of them are assumed degrading and four are not. This degradation distribution assumption is due to a study done that is related to the structural wire bonding on the device used, which leads the current to take less resistive paths and make degradation effect on 11 wire-bonds rather being on all of them. Which could give the possibility to assume degradation in 11 wire-bonds. Also it is assumed that the rate of degradation for the 11 damaged wire-bonds is the same.

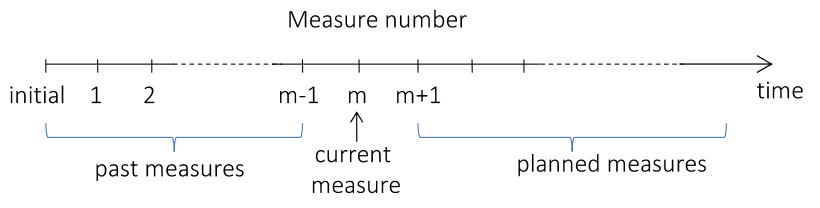

Fig.7: Vce measurements instants

$$
\Delta R(a)_{15 W}=R\left(a_{m}\right)_{15 W}-R\left(a_{0}\right)_{15 W}
$$

$R\left(a_{0}\right)_{15 W}$ and $R\left(a_{m}\right)_{15 W}$ represents respectively the initial resistance of the 15 wire-bonds and the resistance of the 15 wirebonds at the $m^{\text {th }}$ measurement, with:

$$
\begin{gathered}
R\left(a_{0}\right)_{15 W}=\frac{R\left(a_{0}\right)}{15} \\
R\left(a_{m}\right)_{15 W}=\frac{R\left(a_{m}\right) * R\left(a_{0}\right)}{4 R\left(a_{m}\right)+11 R\left(a_{0}\right)}
\end{gathered}
$$

The resistances $R\left(a_{0}\right)$ and $R\left(a_{m}\right)$ are calculated from $\Delta V_{C E}$ using Eq.5-8, the parameter $a_{m}$ is calculated after inverting Eq. 4 and then used to compute $K_{m}$ using Eq.9

$$
K_{m}=\frac{a_{m}-a_{m-1}}{n_{m}-n_{m-1}}
$$

Using the average value $(\bar{K})$ of the $K_{m}$ values $\left(K_{1}, \ldots, K_{m}\right)$, in Eq. 10 gives the predicted values of radius contact $a_{n}$ until the crack propagation completes.

$$
a_{n}=\bar{K} n+a_{0}
$$

where $\bar{K}$ is the average crack propagation rate. "n" represents the number of cycles for the prediction process at each planned measurement $\mathrm{m}$.

By introducing the predicted values of $a_{n}$ in Eqs.5-8, the values of $\Delta V_{C E} / V_{C E, \text { ini }}$ are calculated.

Which means that at each new measurement $\mathrm{m}$, a prediction curve that predicts $\Delta V_{C E} / V_{C E, \text { ini }}$ is formed. This prediction curve stops when the contact area radius " $a_{n}$ " reaches zero, that means lift-off.

For instance, for $\mathrm{m}=1, \Delta V_{C E} / V_{C E, \text { ini }}$ is measured at 8 kilocycles and is equal to $0.16 \%$ as shown in Fig.8. This allows to calculate the area contact radius $a_{1}$. Then, by having $a_{0}$ and $a_{1}$, the first crack speed rate $K_{1}$ is calculated and assumed as constant for predicting the remaining radiuses until "a" reaches zero

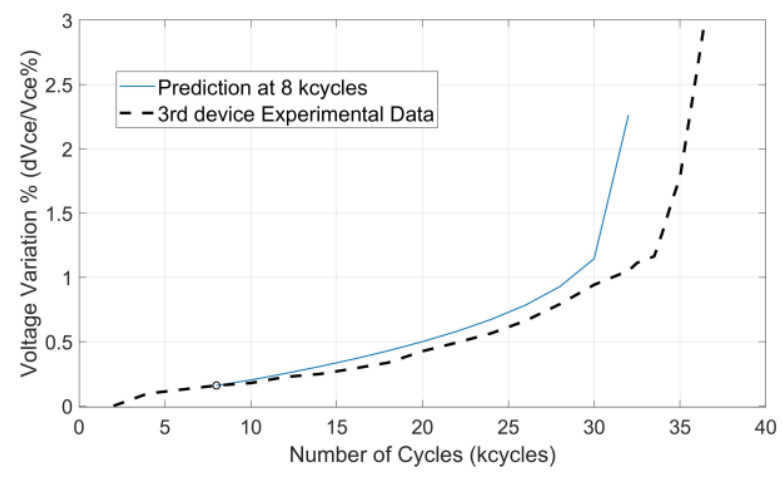

Fig.8: Voltage variation percentage of the first prediction at 8 k.cycles compared to Experimental data

The predicted values of radiuses are reinserted in Eqs.5-8 to calculate the predicted evolution of the curve $\Delta V_{C E} / V_{C E, \text { ini }}$. The first prediction can be observed in Fig.8. By repeating the previous process for several measurements and plotting the predicted $\Delta V_{C E} / V_{C E \text {,ini }}$, Fig.9 could be observed.

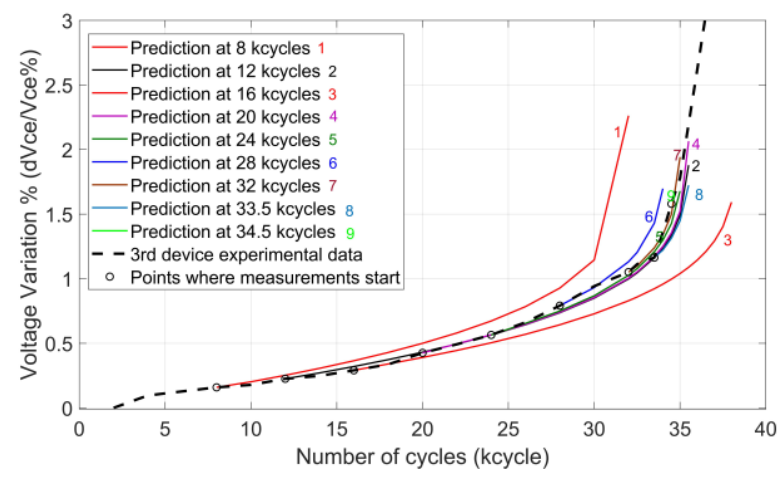

Fig.9: Prediction of the relative increase in $V_{C E}$ for IGBT device under the conditions $\Delta T=110^{\circ} \mathrm{C} \&$ ton $=3 \mathrm{sec}$

Fig.9 can show the predicted curves for $\Delta V_{C E} / V_{C E \text {,ini }}$ at several numbers of cycles. The experimental results in Fig.9 refer to a device aging under $\Delta T=110^{\circ} \mathrm{C}$ and $t_{\text {on }}=3 \mathrm{sec}$. The experimental data used are filtered with an average moving filter on 3 consecutive points.

As it can be observed in Fig.9, the prediction was done to estimate the remaining $\Delta V_{C E} / V_{C E \text {,ini }}$ curve at each step of measurement. The conventional failure criteria assumption on the collector-emitter voltage indicator is when the $\Delta V_{C E} / V_{C E \text {,ini }}$ reaches a $5 \%$ increase. However, it is important to note that this threshold is quickly achieved in the last part of aging, which is wire-bond lift-off (zone 2 Fig.3). In Fig.9, the experimental data represented by the dashed black curve is used to validate the 
predictions. The first and third predictions (at 8 kcycles and 16 kcycles) are less acceptable than the rest of the curves. This is due to the primary construction of the degradation propagation rate. However, this rate is then tuned after each new measurement due to computing the mean of crack propagation rates, and as observed for the remaining six predictions, the estimated $\Delta V_{C E} / V_{C E \text {,ini }}$ is close to the experimental observation and can be relied on.

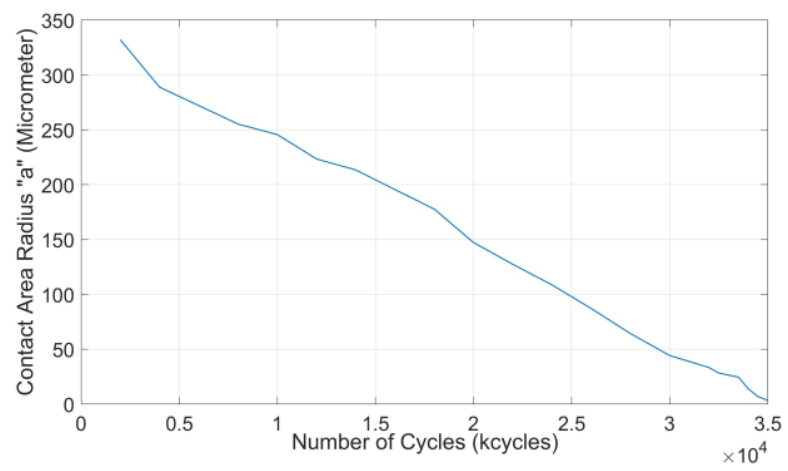

Fig.10: Contact area radius measured $\left(a_{m}\right)$ decrease with the number of cycles for the aging tests done under $\Delta T=110^{\circ} \mathrm{C}$ and $t_{\text {on }}=3 \mathrm{sec}$

At each measurement done a new contact area radius is computed. Fig. 10 presents the radius decrease variation with cycles. It is shown that the variation of radius $a$ with cycles is almost linear. This verifies the assumption of linearity used in crack propagation.

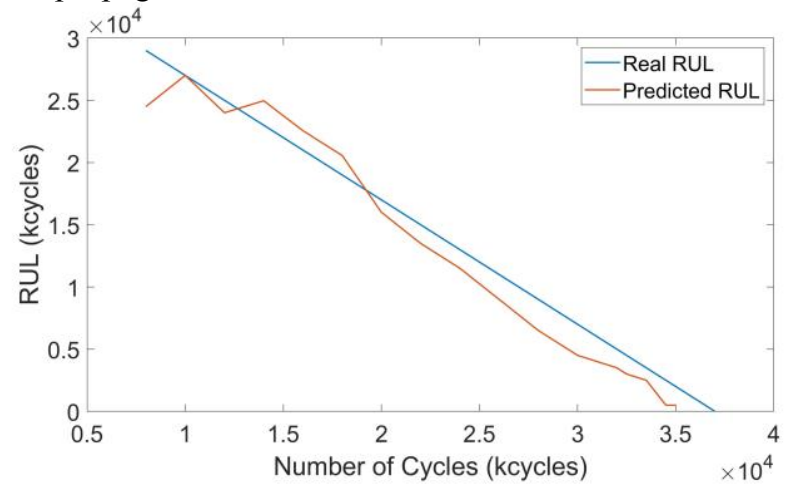

Fig.11: Predict RUL change when applied on aging tests done under the test condition $\Delta T=110^{\circ} \mathrm{C}$ and $t_{\text {on }}=3 \mathrm{sec}$

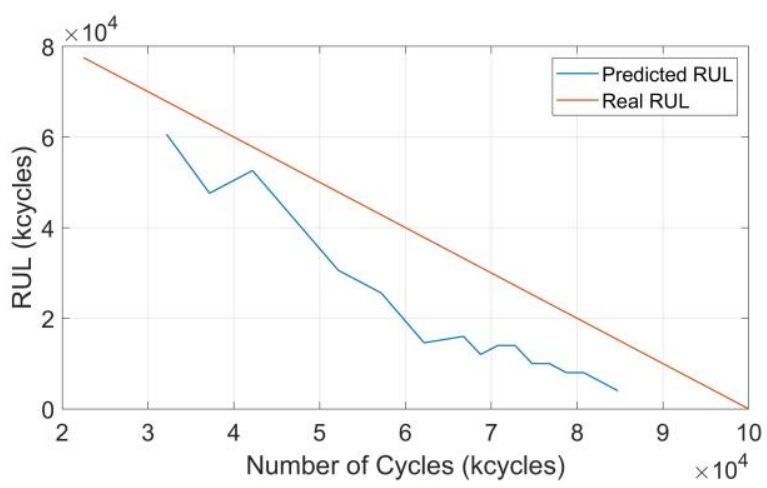

Fig.12: : Predict RUL change when applied on aging tests done under

$$
\text { the test condition } \Delta T=90^{\circ} \mathrm{C} \text { and } t_{o n}=3 \mathrm{sec}
$$

Fig. 11 and Fig. 12 present the predicted RUL and Real RUL change with the number of cycles for the two different test conditions applied in this study. They start where the first prediction curve took place, except for Fig.12 the curve starts from $32 \mathrm{k} . c y c l e s$ due to the errors observed in the experimental data at the first $30 \mathrm{k}$.cycles. The values of predicted RULs could be also verified in Fig.9 and Fig. 11 where the RUL in this study presents the wire-bond's lift-off.

As shown in Fig.12, the predicted RUL from 13 to 18 kilocycles is overestimating the real RUL. The prediction curves are not very reliable. However, after 18 kcycles the RUL is being underestimated and near to the real RUL curve. In both figures, the predicted RUL underestimates the failure which could mean that no additional costs the company will pay to failure consequences.

One way to assess the model robustness is by applying it to different stress conditions. For that, the physical model (including its parameters) is also applied to data aged in different conditions.

The test conditions used in Fig. 13 are for an aging test under $\Delta T=90^{\circ} \mathrm{C}$ and $t_{\text {on }}=3 \mathrm{sec}$. As expected, these tests last much more than tests under $\Delta T=110^{\circ} \mathrm{C}$. The lifetime for tests at $\Delta T=90^{\circ} \mathrm{C}$ reaches around 100 kcycles whereas the lifetime of the tests at $\Delta T=110^{\circ} \mathrm{C}$ reaches around 35 kcycles. As observed in Fig. 13 the first and second curves are far from the experimental curve. This is due to high variations in data measurements as observed at first 30 kcycles. Then, the prediction curves start to trend upward as the experimental curve.

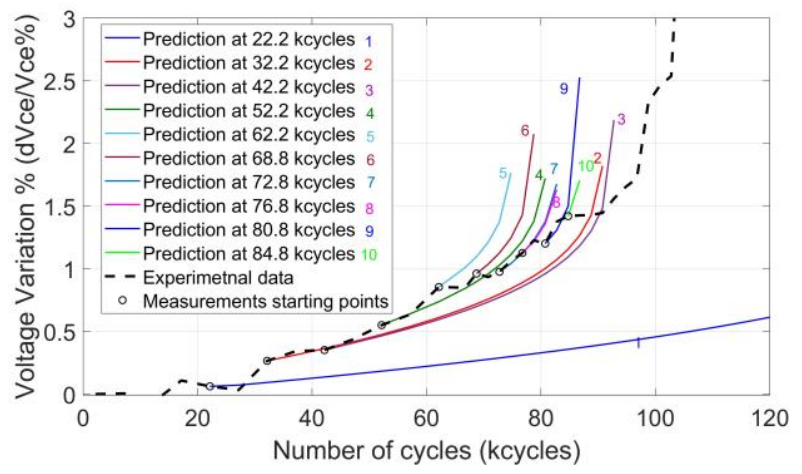

Fig.13: Prediction of the relative increase in $V_{C E}$ for IGBT device under the conditions of $\Delta T=90^{\circ} \mathrm{C} \&$ ton $=3 \mathrm{sec}$

\section{Conclusion}

In this study, a physics-based analytical model is built to relate crack propagation to $V_{C E}$. Since $V_{C E}$ is monitored in realtime operation, then the crack length at each measurement can be estimated. Then a crack rate of propagation is computed and used to predict the crack lengths until the crack is completed and lift-off is achieved, assuming a linear crack propagation. These predicted crack lengths give the predicted collector-emitter voltage variation using the model built. The rate of crack 
propagation is averaged at each new measurement and used for the next prediction. The predicted $V_{C E}$ identifies the end of zone 1, which forms $80 \%$ of the total lifetime of the IGBT semiconductor device. The importance of this work lies under the online prediction ability of the important part of the lifetime in the IGBT; it could be improved to predict the whole life.

\section{Appendix}

The metallization resistance is formulated considering Fig.14.

Considering $J_{0}$ as the vertical current density in the die, $I_{1}$ the total current from die under the contact $\left(I_{1}=J_{0} \pi a^{2}\right)$ and $I_{2}$ the total current outside the contact $\left(I_{2}=J_{0} \pi\left(b^{2}-a^{2}\right)\right), a$ is the radius of the contact area, and $b$ is the outer radius of the metallization. The whole current is $I=I_{1}+I_{2}$.

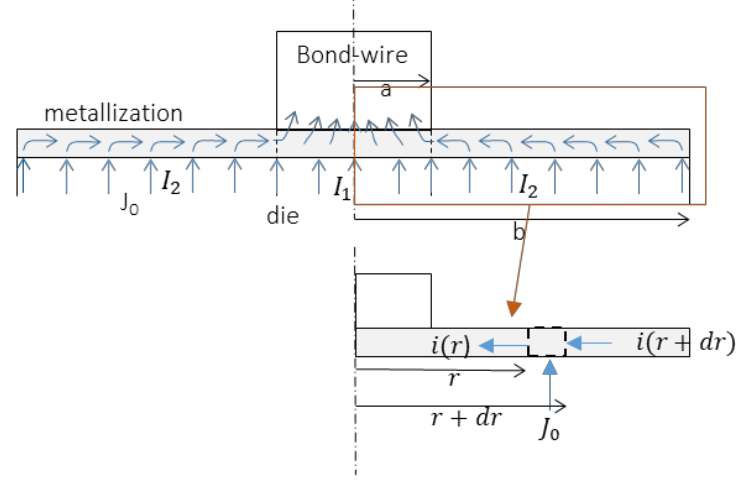

Fig.14: Current distribution study in the metallization passing from Die to wire-bond.

One can observe that the current in the metallization $i(r)$ is negative in the radial oriented axis but the current variation $d i / d r$ at radius $r$ is positive and given by:

$$
d i / d r=J_{0} 2 \pi r d r
$$

Eq.11 is integrated from $r$ to $b$ knowing that $i(b)=0$. The following equation is formed:

$$
i(r)=-\pi J_{0}\left(b^{2}-r^{2}\right)
$$

Where $i(r)$ is the lateral current in the metallization, the negative sign indicates that it is flowing in the reverse direction of the radial oriented axis. Let $j(r)$ be the lateral current density in the metallization at $r$ :

$$
j(r)=\frac{i(r)}{2 \pi r t}=-\frac{1}{\rho} \frac{d V}{d r}
$$

where $\rho$ is the electrical resistivity of the material, and $t$ is the thickness of the metallization. By using Eqs.12 \& 13 the Eq.14 is obtained:

$$
d V=\frac{\rho J_{0}}{2 t} \frac{b^{2}-r^{2}}{r} d r
$$

Thus, after integration from $a$ to $b$ :

$$
V(b)-V(a)=\frac{\rho J_{0}}{2 t}\left(b^{2} \ln \frac{b}{a}-\frac{1}{2}\left(b^{2}-a^{2}\right)\right)
$$

The equivalent resistance of metallization, seen between $r=a$ and $r=b$, can be written:

$$
R_{\text {met. }}=\frac{V(b)-V(a)}{I_{2}}
$$

Then,

$$
R_{\text {met. }}=\frac{\rho}{2 \pi t}\left(\frac{b^{2}}{b^{2}-a^{2}} \ln \frac{b}{a}-\frac{1}{2}\right)
$$

\section{References}

[1] S. S. Manson, thermal stress law-cycle fatigue. New York: McGraw-Hill, 1966.

[2] L. Yang, P. A. Agyakwa, and C. M. Johnson, "Physics-offailure lifetime prediction models for wire bond interconnects in power electronic modules," IEEE Trans. Device Mater. Reliab., vol. 13, no. 1, pp. 9-17, 2013.

[3] N. Dornic et al., "Stress-Based Model for Lifetime Estimation of Bond Wire Contacts Using Power Cycling Tests and Finite-Element Modeling," IEEE J. Emerg. Sel. Top. Power Electron., vol. 7, no. 3, pp. 1659-1667, 2019.

[4] N. Degrenne and S. Mollov, "Diagnostics and Prognostics of Wire-Bonded Power Semi-Conductor Modules subject to DC Power Cycling with Physically-Inspired Models and Particle Filter," Phme 2018, pp. 1-10, 2018.

[5] K. Hu et al., "Cost-Effective Prognostics of IGBT Bond Wires with Consideration of Temperature Swing," IEEE Trans. Power Electron., no. December, pp. 1-1, 2019.

[6] T. Sreenuch, A. Alghassi, S. Perinpanayagam, and Y. Xie, "Probabilistic Monte-Carlo Method for Modelling and Prediction of Electronics Component Life," Int. J. Adv Comput. Sci. Appl., vol. 5, no. 1, pp. 96-104, 2014.

[7] A. Alghassi, "Prognostics and Health Management of Power Electronics," CRANFIELD UNIVERSITY, 2016.

[8] Z. Liu, W. Mei, X. Zeng, C. Yang, and X. Zhou, "Remaining useful life estimation of insulated gate biploar transistors (IGBTS) based on a novel volterra K-nearest neighbor optimally pruned extreme learning machine (VKOPP) model using degradation data," Sensors (Switzerland), vol. 17, no. $11,2017$.

[9] M. Ciappa, "Selected failure mechanisms of modern power modules," Microelectron. Reliab., vol. 42, no. 4-5, pp. 653 667, 2002.

[10] I. Application, P. Under, T. H. E. Patent, and C. Treaty, "I I 1 1 I I," Quantum, no. 12, p. 16, 2015.

[11] J. Zhao et al., "A study on the effect of microstructure evolution of the aluminum metallization layer on its electrical performance during power cycling," IEEE Trans. Power Electron., vol. 34, no. 11, pp. 11036-11045, 2019.

[12] M. Arab, S. Lefebvre, Z. Khatir, and S. Bontemps, "Experimental investigations of trench field stop IGBT under repetitive short-circuits operations," PESC Rec. - IEEE Annu. Power Electron. Spec. Conf., no. 1, pp. 4355-4360, 2008.

[13] S. Pommier, S. Pietranico, M. Berkani, Z. Khatir, S. Bontemps, and E. Cadel, "Effect of die metallization layer ageing in the case of power semiconductor devices," Eur.J. Electr. Eng., vol. 14, no. 5, pp. 569-585, 2011. 
[14] R. Holm, Electric Contacts. Berlin, Heidelberg: Springer Berlin Heidelberg, 1967. 